\title{
Modeling and Simulation of Contention-Collision Cancellation Mode Type IV Star Network*
}

\author{
Lijun Sun, Yunxian Du \\ School of Information Science and Technology, Qingdao University of Science and Technology, Shandong Province, China \\ sun_lijun@yahoo.com.cn, duyunx846@163.com
}

\begin{abstract}
Contention-Collision Cancellation(C-CC) mode is an access control mode in star network. According to the working mechanism of star network, C-CC access control mode was classified six types of system model. The mathematical model of type IV system model is among the most complex at a very high computational cost so that its numerical analysis of the real network environment is hard to realized. In order to evaluate the performance of this type networks, we discuss the process of constructing the star network and implementing type IV system model using by OPNET modeler. Firstly, we compare the simulation result to the theoretical result to ensure the accuracy of the simulation model. Then in conditions close to the real network environment, we get three important performance parameters: packet delay, the queue size and throughout of central node. Finally, we analyze the impact on network performance of the value of service time in different load conditions, and provide a basis for setting reasonable length of data packet and ACK packet, thereby optimizing the network performance.

Index Terms - Simulation modeling; Star network; ContentionCollision Cancellation access control mode.
\end{abstract}

\section{Introduction}

In the star network communication, contention-collision cancellation (C-CC) access mode is an important access control mode. According to the working mechanism of star network[1], C-CC access control mode was classified six types of system model[2]. We have made a great breakthrough in the mathematical modeling and mathematical analysis of these six types system model[3-8]. The mathematical model of type IV system model is among the most complex at a very high computational cost so that its numerical analysis of the real network environment is hard to be realized. We have done the numerical calculation in Ref.[6], but we only set the service time $f$ as two when carrying the experiment. This is inconsistent with the actual network environment. It is still not perfect that make the service time increased to 8 though introducing a new data storage structure in Ref.[9]. So it is necessary to use simulation experiment to get the result when $f$ is larger. In order to evaluate the performance of this type networks, we discuss the process of constructing the star network and implementing type IV system model using by OPNET modeller. Firstly, we compare the simulation result to the theoretical result to ensure the accuracy of the simulation model. Then in conditions close to the real network environment, we get three important performance parameters: packet delay, the queue size and throughout of central node.

\footnotetext{
* This work is partially supported by NSF Grant \#60902034.
}

Finally, we analyze the impact on network performance of the value of service time in different load conditions, and provide a basis for setting reasonable length of data packet and ACK packet, thereby optimizing the network performance.

For convenience, let's introduce the working principle of star network briefly. Here we consider the star network with $N$ user terminals and only 1 switch channel in central node (Fig. 1), $N>>1$. If a user terminal generates a new data unit, it put the data unit into its own buffer and doesn't generate new data units until the buffer is free. Once a new data unit is generated, it will be sent out immediately (i.e. ask for service). If more than one data unit ask for service simultaneously, collision will happen. The central node selects one data unit randomly and switches it, and the remainders are all eliminated. If the selected data unit is switched correctly (i.e. the service succeeds), its original terminal will receive a response unit ACK. Otherwise, the original terminal will receive a NAK and the data unit will ask for the service again. The eliminated data units in collision will ask for the service again after a constant period of time. Generally, this constant period of time is called overtime, which is from the data unit being sent out to an ACK arriving.

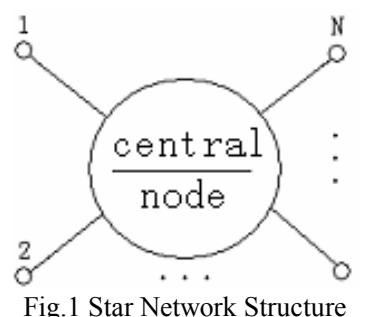

\section{OPNET Simulation and Modeling}

A. C-CC access mode type IV system model

In the system of $\mathrm{C}$-CC access control mode, we treat central node as a server and the data units generated by user terminal as customers. The customer's primary request is stochastic, and the secondary request is at a constant interval. If the service requirement is successful, the customer receives service and departs the system, if the service requirement is failure, the customer abandons the service right and ask for service anew until is served correctly.

In this paper, we use the OPNET simulation platform complete the simulation for the type IV system model. 


\section{B. Network Domain and Node Domain}

The OPNET modelling works in three different environments which is also known as modeling domain, such as network domain, node domain and process domain.

The role of the network domain is to define the topology of the communication network. The node domain configures and models the connected communication equipment. Process domain describes the protocol with finite state machine.

This star network is formed by one central node and $N$ user terminals as shown in TABLE I. The modules of network domain and node domain are shown in the Fig.2.

TABLE I The mapping of the star network to the OPNET model

\begin{tabular}{|c|c|c|}
\hline $\begin{array}{c}\text { Network } \\
\text { domain }\end{array}$ & \multicolumn{2}{|c|}{$\begin{array}{c}\text { Star } \\
\text { topology }\end{array}$} \\
\hline $\begin{array}{c}\text { Node } \\
\text { domain }\end{array}$ & $\begin{array}{c}\text { 1 central node (with } 40 \\
\text { pairs of transceiver) }\end{array}$ & $\begin{array}{c}40 \text { user terminals (with 1 } \\
\text { pair of transceiver) }\end{array}$ \\
\hline $\begin{array}{c}\text { Process } \\
\text { domain }\end{array}$ & server process & user process \\
\hline
\end{tabular}

\section{Network Domain and Node Domain modeling}

User node of the user process is responsible for producing, sending, receiving and processing of data unit. It can send the generated data unit, process the data received from the central node, and statistics the delay time of package. Each free user generates at most one data unit in a slot with probability $p_{c}$.

The server process of central node is responsible for selecting randomly 1 data unit from requests and sending it.

The state transition diagram of user process and server process is show as Fig.3-Fig.4.

\section{Simulation Results and Performance Evaluation}

We set the transmission rate of network is $100 \mathrm{Mbps}$.

The simulation time is 0.01 second.

\section{A. Comparison of the Simulation results with the Theoretical Value}

In ref.[5], mathematical modeling and simulation for type IV have been completed. In order to compare with the theoretical results acquired in ref[5], in this paper $N=40$ 、 $p_{c}=0.1 、 p_{s}=0.5$ and $f=2$. Dependence of the mean number of the customers and the mean waiting time on time are shown in Fig.5 and Fig.6. In Fig.5, at the beginning of the simulation all user terminals generate customer with a certain probability, so the average number of the customer is increased rapidly. When the system reach the steady state the average number of the customer is approximately equal to 37.In Fig.6, with the increasing of generated customers, the collision may happen so the waiting time become longer, when the system reach the steady state, the mean waiting time is approximately equal to 21us.

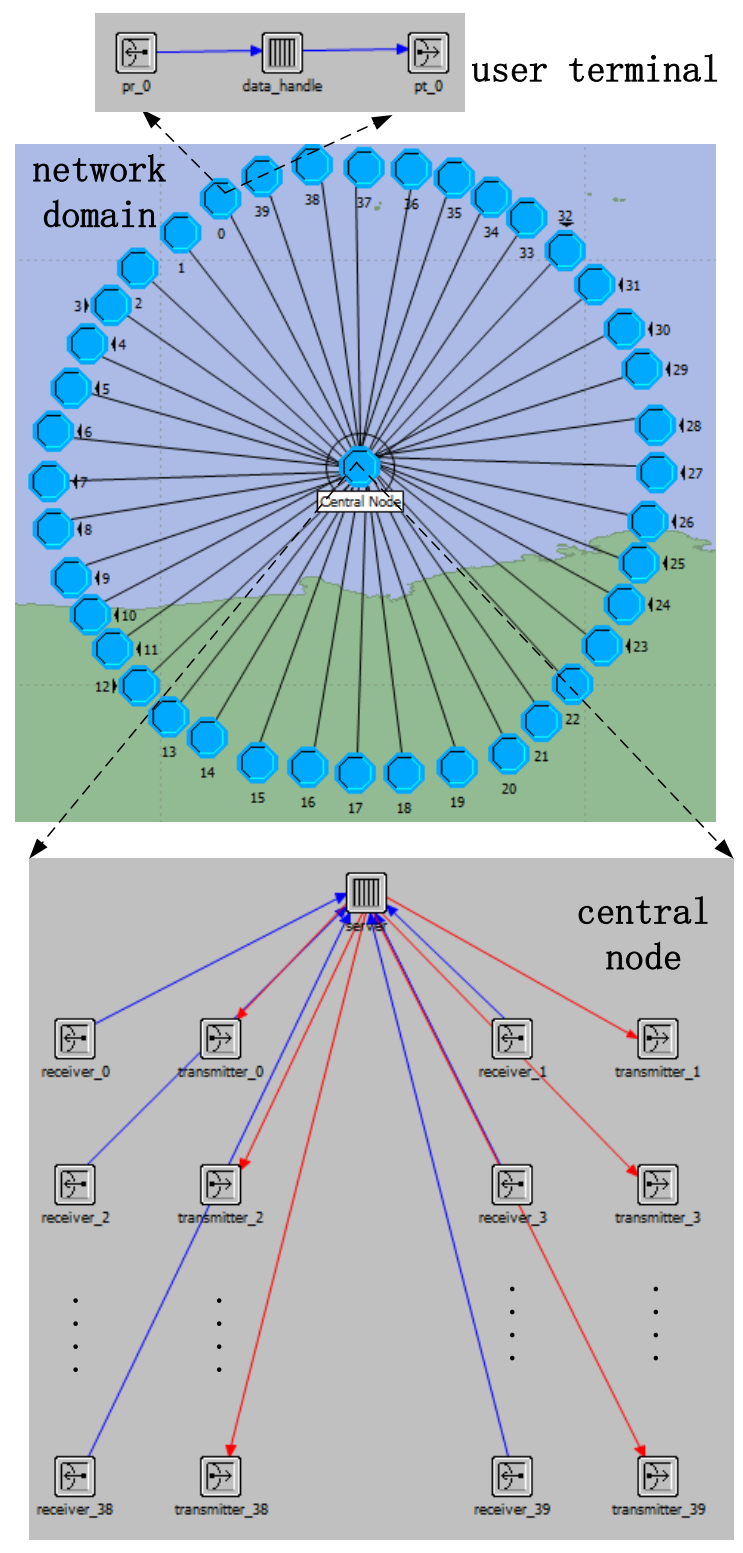

Fig.2 Network domain and node domain

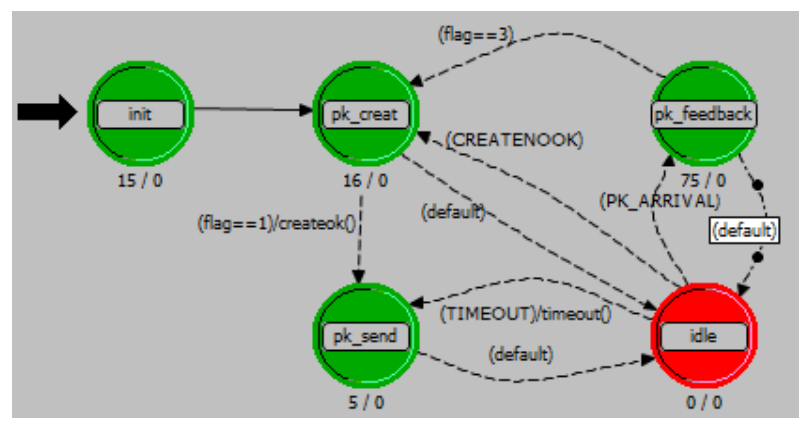

Fig.3 State transition diagram of user process 


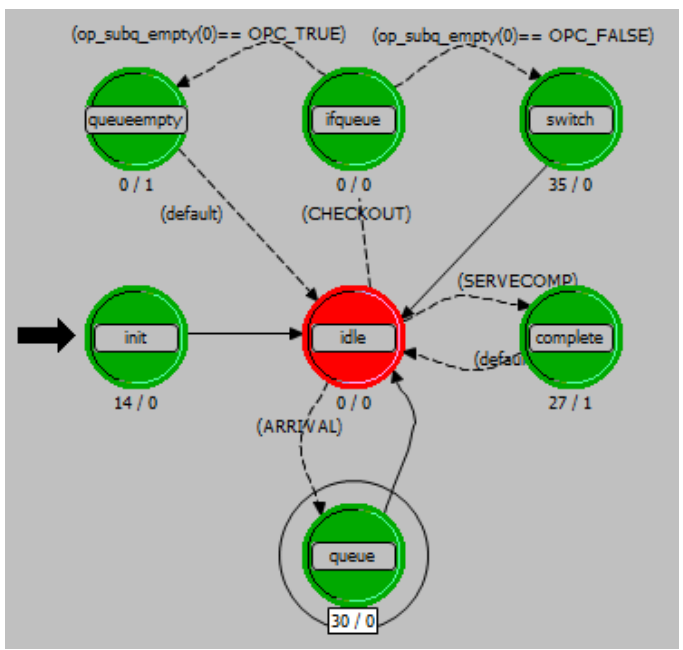

Fig.4 State transition diagram of server process

As shown in Fig.5 and Fig.6, the results of the mathematical modeling and the simulation are very close. From the simulation it is proved that the mathematical modeling in ref[6] is right.

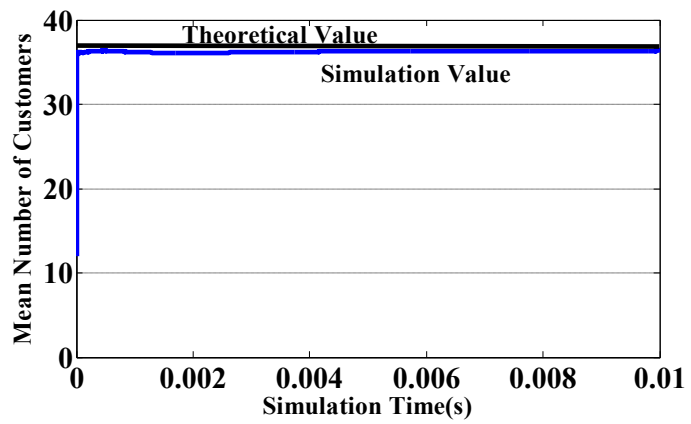

Fig.5 The comparison between the simulation value and the theoretical value of the mean number of customer

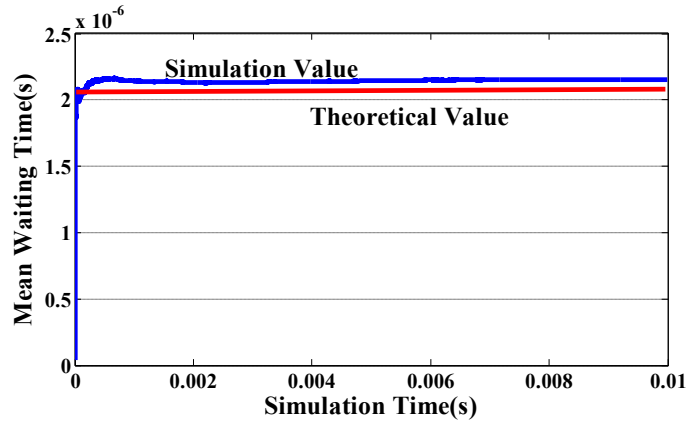

Fig.6 The comparison between the simulation value and the theoretical value of the mean waiting time

\section{B. Analysis of Simulation Results}

The dependence of the mean waiting time and the average number of the customers on simulation time with different $p_{c}$ are shown in fig.7 and fig.8. The simulation selects $N=40$, $p_{s}=0.95$ and $f=20$. If $p_{c}<0.04$, the speed of curves descends fast along with the decreasing of $p_{c}$.
In fig. $7, p_{c}=0.001$, when system reach the steady state, the number of the customers is 6 and when $p_{c}=0.02$ it is increased to 33. After $p_{c}$ increases to 0.04 the number is 37 and it is almost the same with the situation $p_{c}=0.1$. It can be summed up that when $p_{c}$ is less than or equal to 0.04 the system is light load. In this situation $p_{c}$ make a great influence on mean number of customers. But when $p_{c}>0.04$, the system is high load, the $p_{c}$ make little influence on mean number of customers, the steady state number is 40 .

In fig.8, If $p_{c}<0.04$, the speed of curves increasing becomes fast along with the increasing of $p_{c}$. When $p_{c}>0.04$ the increasing of mean waiting time is slow. It can be seen that Fig. 8 is consistent with the Fig.7, but with the increasing of $p_{c}$ the mean waiting time increase slowly.

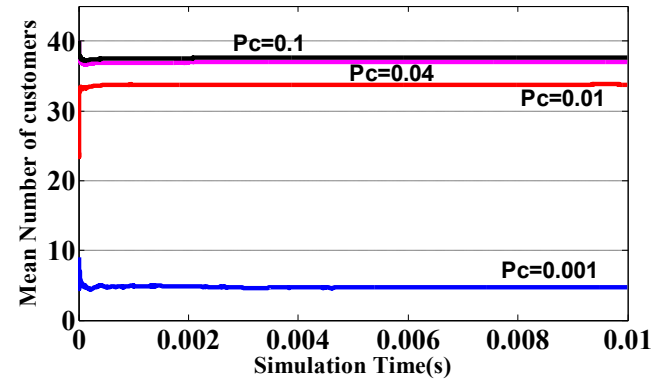

Fig.7 The comparison of the mean number of customer for different $p_{c}$

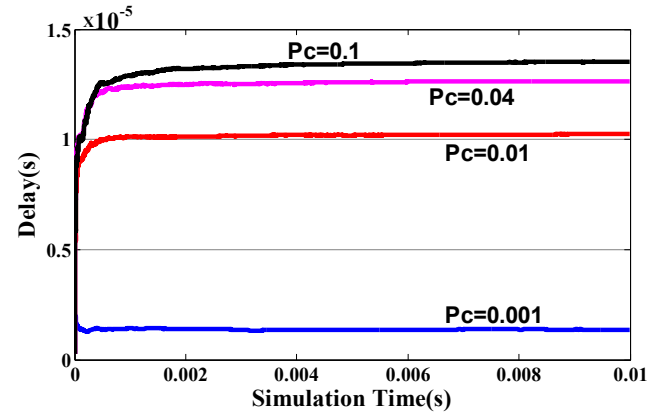

Fig.8 The comparison of the delay for different $p_{c}$

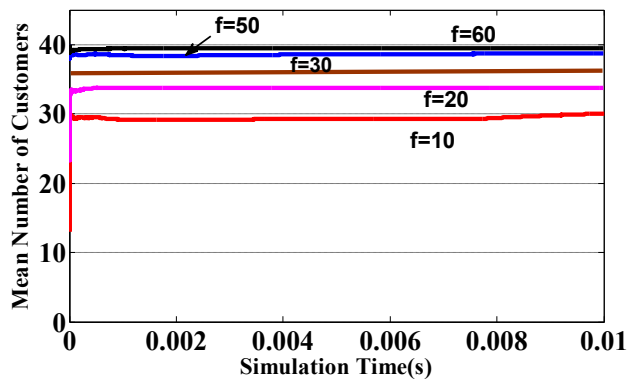

Fig.9 The comparison of the mean number of customer for different $f$

In ref.[6], $f$ is not only the length of time of service, but also the length of the timeout period, which actually starts at the time when the source terminal send data packet, and ends at the time the reception of ACK. ACK packet size can be 
fixed but packet size is depending on $f$. In order to observe different $f$ impact on network performance, $p_{c}=0.01, p_{s}=$ $0.95, N=40$ is selected, and $f$ is equal to $10,20,50$ and 60 . In Fig. 9-10, the average number of the customers and the throughput are shown.

As shown in Fig.9, the number of customers is straight up at the beginning of the simulation. Soon the number of customers is stabilized at a value. When $f$ is increased from 10 to 20 , the average number of customers increases fast. When $f$ increases by 20 to 60 , the average number of customers increases slowly, and finally close to the number of user terminals. In Fig.10, when system reach the steady state the throughput of the central code increases fast when $f$ increase from 2 to 20 . While $f$ increases from 20 to 50 , the throughput increases relatively slowly. When $f=30$ the throughput curve almost coincides with the situation $f=50$, which shows that the system reached saturation throughput. Thus we obtained with the increase of the $f$, the length of the packet increased. At the same time the number customer retention, the throughput is also increased. Consolidated throughput and delay factors, in current network environment, $f=30$ is more appropriate.

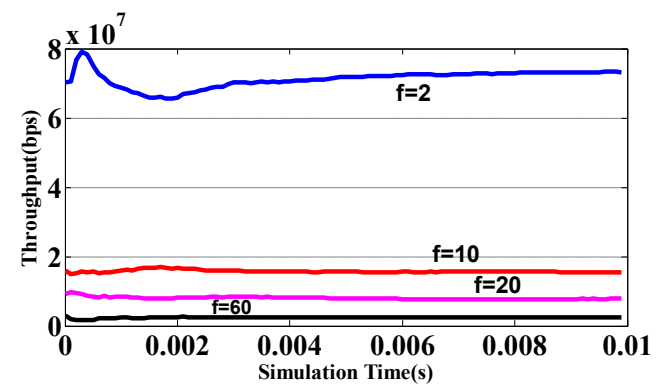

Fig.10 The comparison of the throughput of the central node for different $f$

Setting $p_{c}=0.001$, run the simulation again to get lighter loads with different $f$, the results are shown in Figure 11-12. The central node throughput increases with the increase of the $f$. When $f=60$ the throughput curve coincides almost the situation $f=70$. In other words, the system reaches the saturation throughput. The delay also increases with the $f$ increase. It is easy to find out when $p_{c}=0.001$ the load is light, the $f$ can be set with a larger value of 60 .

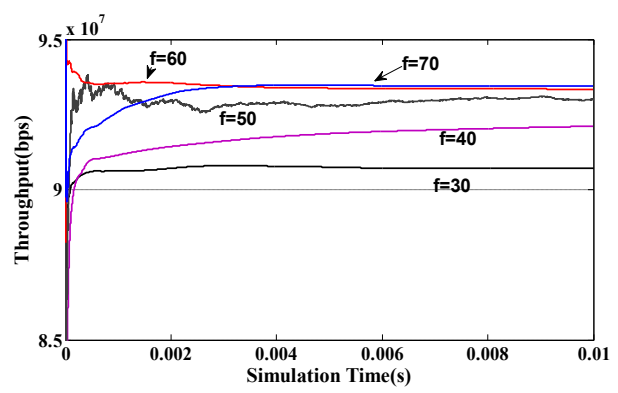

Fig.11 The comparison of the throughput of the central node for different $f$ when $p_{c}=0.001$

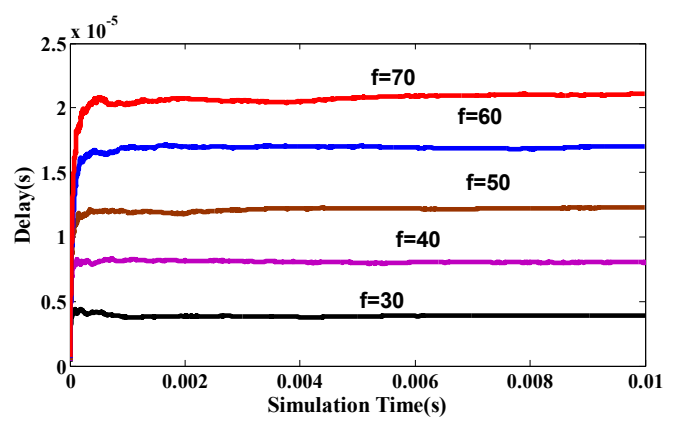

Fig. 12 The comparison of the delay for different $f$ when $p_{c}=0.001$

\section{Acknowledgment}

This work is supported by National Natural Science Foundation of China (60902034) and promotive research fund for excellent young and middle-aged scientists of Shandong Province (BS2011DX009).

\section{References}

[1] T.Saito et al, "The star type LAN having multiple access capability," J. Inst. Electron. Commun. Eng. Jpn, vol. B-I, JB-B-1,no. 1, pp. 9-16, 1990

[2] Zhaoyi. Lu, "The performance judgment for the 1st type system model in contention-collision elimination access mode," Acta Electron. Sinica, vol. 23, no. 9, pp. 115-117, 1995.

[3] Zhaoyi Lu, Lijun Sun, "Mathematical model for access mode of contention-collision cancellation in a star LAN," Journal of Electronics, vol. 21, no. 1, pp. 37-47, 2004

[4] Zhaoyi Lu, Mai Lu, Dongfeng Zhao, et al, "On the 2nd model of contention-collision elimination access mode in star LAN," Applied Math. Modeling, vol.27, no. 11, pp. 899-911, 2003.

[5] Zhaoyi Lu, et al, "A special random selective services queuing model for access to a star LAN," Applied Math. Modeling, vol. 21,no. 1, pp. 15-20, 1997.

[6] Zhaoyi Lu, Lijun Sun, Mai Lu, et al, "The mathematical modeling for the Contention-Collision Elimination access mode of the 4th system model to a multiple star LAN," Applied Math Modeling, vol. 30, no. 44, pp. 367$385,2006$.

[7] Zhaoyi Lu, Jianshu Zhang, Mai Lu, "The mathematical modeling for the access mode of the 4th System Model to a Multiple Star LAN," Applied Math. Modeling, vol. 23, no. 3, pp. 231-239, 1999.

[8] Zhaoyi Lu, Xingmei Yang, Chao Wang, "The mathematical modeling for the Contention-Collision Elimination access mode of the 4th system model to a multiple star LAN," Acta Electronica.Sinica,vol.35,no.10,pp.1817-1822,2007.

[9] Lijun Sun. "Performance Analysis of the Type IV System Model of Contention-Conllision Cancellation Access Mode": 2010 3rd International Conference on Computer and Electrical Engineering,Chengdu,2010[C]. 